\title{
ASPECTOS FARMACOLÓGICOS DA Matricaria Recutita (CAMOMILA) NO TRATAMENTO DO TRANSTORNO DE ANSIEDADE GENERALIZADA E SINTOMAS DEPRESSIVOS
}

\author{
PHARMACOLOGICAL ASPECTS OF Matricaria Recutita (CHAMOMILE) IN THE \\ TREATMENT OF GENERALIZED ANXIETY DISORDER AND DEPRESSIVE \\ SYMPTOMS
}

\section{Susana Silva Lima1', Romério de Oliveira Lima Filho², Guilherme Antônio Lopes de Oliveira³}

1 - Discente do Curso Bacharelado em Enfermagem na Cristo Faculdade do Piauí - CHRISFAPI

2 - Discente do Curso Bacharelado em Biomedicina na Universidade Federal do Piauí - UFPI

3 - Docente da Cristo Faculdade do Piauí - CHRISFAPI, Doutor em Biotecnologia pela RENORBIO-UFPI

Autor para correspondência: susi.lima98@gmail.com

\section{RESUMO:}

A ansiedade é um sentimento intrínseco do ser humano que serve como defesa a possíveis estressores. Quando situações de preocupações se tornam mais frequentes, pode-se pensar em uma ideia de ansiedade como um transtorno psiquiátrico. Percebe-se que os transtornos de ansiedade e os sintomas depressivos estão cada vez mais prevalentes, sendo de suma importância o estudo acerca do seu tratamento e abordagens terapêuticas. Reflete-se, então, sobre a possibilidade da utilização da Matricaria recutita (camomila) para a atenuação destes sintomas, visto que é uma planta amplamente utilizada na medicina popular por sua atividade calmante. Estudar acerca dos benefícios e da abordagem fitoterapêutica com a Matricaria recutita (camomila) para o TTO do TAG e na redução dos sintomas depressivos e discorrer sobre suas propriedades. Trata-se de uma revisão bibliográfica com análise de conteúdo e abordagem qualitativa. A pesquisa evidenciou que o extrato de camomila produziu uma redução clinicamente significativa em pacientes com TAG moderado a grave e com sintomas depressivos, mostrando-se eficaz sobretudo por sua baixa toxicidade e boa tolerabilidade. Observou-se com o presente estudo que a Matricaria recutita tem destaque relevante, apresentando resultados satisfatórios no controle da ansiedade, bem como na redução dos sintomas depressivos, além de chamar a atenção por sua baixa toxicidade, o que representa importante característica para a produção de um fitoterápico, de forma a abranger seu uso para além do empirismo empregado pela cultura popular, o que proporcionaria maior aproveitamento de sua propriedades farmacológicas.

Palavras-chave: Fitoterapia. Matricaria recutita. Ansiedade. Depressão.

\section{ABSTRACT}

Anxiety is an intrinsic feeling of the human being that serves as a defense to possible stressors. When situations of concern become more frequent, one may think of an idea of anxiety as a psychiatric disorder. It is noticed that anxiety disorders and depressive symptoms are increasingly prevalent, being of paramount importance the study about their treatment and therapeutic approaches. The use of Matricaria recutita (chamomile) to attenuate these symptoms is considered, since it is a plant widely used in folk medicine for its calming activity. To study the benefits and the phytotherapeutic approach with chamomile would bring to the TTO of the TAG and to reduce the depressive symptoms and to discuss its properties. This is a bibliographical 
review with content analysis and qualitative approach. The research evidenced that the extract of chamomile produced a clinically significant reduction in patients with moderate to severe TAG and with depressive symptoms, proving to be effective mainly due to its low toxicity and good tolerability. It was observed with the present study that Matricaria recutita has a relevant prominence, presenting satisfactory results in the control of anxiety, as well as in the reduction of depressive symptoms, besides calling attention to its low toxicity, which represents an important characteristic for the production of a phytotherapic, in order to cover its use beyond empiricism employed by popular culture, which would provide greater use of its pharmacological properties.

Keywords: Phytotherapy. Matricaria recutita. Anxiety. Depression.

\section{INTRODUÇÃO}

A ansiedade é um sinal apto do ser humano de estar em alerta quando há perigo iminente. Esta capacita alguém a tomar certas medidas que o faça saber lidar com a ameaça desconhecida e conflituosa. Essa sensação, quando em níveis baixos, serve para advertir ameaças como lesões corporais, possíveis punições ou frustrações de necessidades sociais (SADOCK; SADOCK, 2017).

Quando o indivíduo se apresenta ansioso em inúmeras situações e evidencia preocupações excessivas na maior parte dos dias, há uma grande probabilidade desta ansiedade não vir a se apresentar apenas como circunstância adaptativa, mas começa a se pensar numa ideia de ansiedade como transtorno psiquiátrico (STAHL, 2014).

Com aumento da frequência da ansiedade generalizada (TAG) e dos sintomas depressivos na clínica, é de suma importância o estudo acerca do seu tratamento (TTO) e suas principais abordagens terapêuticas. O tratamento mais recorrente destes sintomas é através do uso de benzodiazepínicos (BZD) devido à sua boa aceitabilidade e tolerabilidade (ANDREATINI; BOERNGEN-LACERDA; ZORZETTO FILHO, 2001).

Porém, há uma grande preocupação em relação aos BZD, pois os indivíduos que utilizam a droga, muitas vezes, utilizam-na de forma inadequada. Muitos pacientes abusam do uso por criarem expectativa de que a medicação irá ajudá-los a resolver seus problemas ou simplesmente pelo prazer de seus efeitos agradáveis que promovem uma maior excitação e motivação para realizar atividades cotidianas (ANDREATINI; BOERNGENLACERDA; ZORZETTO FILHO, 2001; LARANJEIRA, 1999).

Observado que o Brasil é reconhecido por sua biodiversidade, torna-se oportuno um modelo de desenvolvimento próprio e autônomo na área de fitoterápicos, pautado no uso sustentavel e racional, promovendo a valorização das práticas tradicionais de uso de plantas medicinais como elementos para a promoção da saúde, conforme preconiza a OMS (BRASIL, 2016).

Dessa forma, reflete-se sobre a possibilidade da utilização de plantas medicinais 
para a atenuação de sintomas depressivos e do TAG. Visto que estas são frequentemente apresentadas como um grande potencial para a origem de novos fármacos devido serem fontes de substâncias bioativas que podem ter grande influência sobre a saúde (SIMÕES, 2007; FAUSTINO; ALMDEIDA; ANDREATINI, 2010).

A camomila (Matricaria recutita L.) por sua vez, é uma planta muito utilizada tanto de forma empírica na medicina tradicional, como na forma de medicamento fitoterápico, através da extração de flavonoides e óleo volátil presentes nos capítulos florais, responsáveis por suas propriedades terapêuticas, com destaque para a ação ansiolítica (BRASIL, 2015).

Observada a elevada incidência do TAG em coocorrência com sintomas depressivos, além dos impactos causados pela terapia medicamentosa convencional desses transtornos, tornou-se oportuno o estudo acerca de quais benefícios a abordagem fitoterapêutica com a Matricaria recutita (camomila) traria para o TTO do TAG e na redução dos sintomas depressivos, discorrendo sobre suas propriedades.

\section{REFERENCIAL TEÓRICO}

\subsection{Transtorno de ansiedade generalizado e transtorno depressivo}

Segundo Sadock e Sadock (2017), o TAG é uma condição comum, sendo o transtorno mais incidente entre os transtornos de ansiedade na população ( 3 a $5 \%)$. Sua causa permanece pouco esclarecida e atinge um contingente heterogêneo. Dentre os principais sintomas estão a tensão muscular, cefaleias, hiperatividade autonômica e distúrbios gastrointestinais. Goodman e Gilman (2005) completam que os sintomas de ansiedade estão comumente associados à depressão.

De acordo com o DSM-V (2014) o transtorno depressivo maior, envolve alterações nítidas no afeto, na cognição e em funções neurovegetativas, podendo ocorrer remissões interepisódicas. O paciente experimenta sintomas como distúrbios do sono e na atividade, falta de energia, sentimentos de culpa, ideação suicida e dificuldade de pensamento. Está associado com alta mortalidade, em boa parte contabilizada pelo suicídio, pode aparecer pela primeira vez em qualquer idade, mas a probabilidade de início aumenta sensivelmente com a puberdade.

Para Silveira (2014), há um alto custo da depressão para a sociedade, sendo comparável às mais graves e incapacitantes doenças crônicas. Ela ainda pode interferir no prognóstico de outras patologias, agravando a doença de base e diminuindo a resposta ao tratamento clínico. 


\subsection{Aspectos gerais da camomila}

A camomila (Matricaria recutita L.) é uma planta muito utilizada tanto na medicina tradicional, como na forma de medicamento fitoterápico, através da extração de flavonoides e óleo volátil presentes nos capítulos florais, responsáveis por suas propriedades terapêuticas, com destaque para a ação ansiolítica. O medicamento fitoterápico é o obtido por processos tecnologicamente adequados, empregando-se exclusivamente matérias primas vegetais, com finalidade profilática, curativa, paliativa ou para fins de diagnóstico, deve-se conhecer sua eficácia e os riscos de seu uso, além da reprodutibilidade e constância de sua qualidade (BRASIL, 2000; BRASIL, 2010; BRASIL, 2015).

Ela é uma das mais citadas para fins medicinais em estudos quanti- qualitativos para uso adulto ou pediátrico, sendo citada em farmacopeias, estudos etnobotânicos, medicina popular, complementar e alternativa. Em 2014, passou a fazer parte da Relação de Medicamentos Fitoterápicos e de Produtos Tradicionais Fitoterápicos na categoria de produto tradicional fitoterápico de registro simplificado (BRASIL, 2015).

O volume 1 da Farmacopeia Brasileira 5. ed. (2010) traz para fins de definições gerais quanto a ação, uso e doses dos medicamentos, sendo essas as constantes do relatório para registro do produto no órgão sanitário, atualizadas mediante revisão bibliográfica nacional e internacional. Quando indicadas nas monografias, as doses representam a quantidade do medicamento usualmente prescrita que tenha eficácia terapêutica para pacientes adultos. A dose considera a farmacocinética e a farmacodinâmica, podendo variar as quantidades e a frequência de administração de qualquer medicamento, exclusivamente sob a responsabilidade exclusiva do prescritor habilitado (BRASIL, 2011; BRASIL, 2010).

\section{METODOLOGIA}

Tratou-se de um estudo de revisão integrativa na literatura, de caráter descritivo e qualitativo acerca da temática.

Utilizaram-se os descritores ansiedade, depressão, fitoterapia e Matricaria recutita indexados e usados de forma associada. Para a coleta de dados foram utilizadas as bases de dados da Biblioteca Virtual em Saúde (BVS), Google Acadêmico, PubMed e literatura física.

Para critérios de inclusão, postularam-se obras publicadas na íntegra em línguas portuguesa e inglesa, datados de 2009 a 2019. Foram excluídos as que não se encaixaram 
nos critérios de inclusão e não englobaram a temática de forma satisfatória.

4. RESULTADOS E DISCUSSÃO

Tabela 1: Estudos acerca da camomila no tratamento do transtorno de ansiedade generalizada e sintomas depressivos

\begin{tabular}{|c|c|c|c|c|}
\hline TÍTULO & AUTORES & ANO & OBJETIVO & METODOLOGIA \\
\hline $\begin{array}{c}\text { Short-term open-label } \\
\text { chamomile (Matricaria } \\
\text { chamomilla L.) therapy } \\
\text { of moderate to severe } \\
\text { generalized anxiety } \\
\text { disorder }\end{array}$ & $\begin{array}{l}\text { Keefe JR, } \\
\text { Mao JJ, } \\
\text { Soeller I, Li } \\
\text { QS, } \\
\text { Amsterdam } \\
\text { J }\end{array}$ & 2016 & $\begin{array}{l}\text { Verificar se o TTO com } \\
\text { extrato de camomila } \\
\text { resultaria em uma } \\
\text { redução significativa nas } \\
\text { classificações de } \\
\text { gravidade do TAG, e } \\
\text { estaria associado a um } \\
\text { evento adverso } \\
\text { favorável e perfil de } \\
\text { tolerabilidade. }\end{array}$ & $\begin{array}{c}\text { Tratamento aberto com extrato de } \\
\text { camomila de grau farmacêutico } \\
\text { 1500mg/dia por até } 8 \text { semanas em } \\
\text { pessoas com TAG moderada a grave. } \\
\text { Controle através das Escala de } \\
\text { Ansiedade de Hamilton, no } \\
\text { Inventário de Ansiedade de Beck e no } \\
\text { Índice Geral de Bem-estar } \\
\text { Psicológico. Eventos adversos e } \\
\text { descontinuação do TTO foram } \\
\text { examinados. }\end{array}$ \\
\hline $\begin{array}{l}\text { MONOGRAFIA DA } \\
\text { ESPÉCIE Matricaria } \\
\text { chamomilla L. (= } \\
\text { Chamomilla recutita (L.) } \\
\text { Rauschert, CAMOMILA }\end{array}$ & BRASIL & 2015 & $\begin{array}{l}\text { Avaliar as atividades de } \\
\text { infusão da planta contra } \\
\text { cepas. }\end{array}$ & $\begin{array}{c}\text { Ensaio comparativo de triagem (CSA) } \\
(4,5 \mathrm{mg} / \mathrm{mL}) \text {, método de difusão em } \\
\text { ágar (AWDM) }(0,4 \\
\text { mg/ poço) e microscopia. }\end{array}$ \\
\hline $\begin{array}{l}\text { Long-Term Chamomile } \\
\text { Therapy of Generalized } \\
\text { Anxiety Disorder: A } \\
\text { Study Protocol for a } \\
\text { Randomized, Double- } \\
\text { Blind, Placebo- } \\
\text { Controlled Trial }\end{array}$ & $\begin{array}{l}\text { Jun J Mao, } \\
\text { Qing S Li, } \\
\text { Irene Soeller, } \\
\text { Kenneth } \\
\text { Rockwell, } \\
\text { Sharon X Xie } \\
\text { and Jay D } \\
\text { Amsterdam }\end{array}$ & 2014 & $\begin{array}{c}\text { Examinar a segurança e } \\
\text { a eficácia em curto e } \\
\text { longo prazo do extrato } \\
\text { de camomila no } \\
\text { Transtorno de } \\
\text { Ansiedade Generalizada } \\
\text { (TAF) }\end{array}$ & $\begin{array}{l}\text { Estudo randomizado duplo-cego com } \\
\text { administração do extrato de camomila } \\
500-1500 \text { mg diários ou placebo por } \\
\text { mais } 26 \text { semanas em } 180 \text { pessoas } \\
\text { com TAG moderado a grave. }\end{array}$ \\
\hline $\begin{array}{l}\text { Chamomile (Matricaria } \\
\text { recutita) May Have } \\
\text { Antidepressant Activity } \\
\text { in Anxious Depressed } \\
\text { Humans - An } \\
\text { Exploratory Study }\end{array}$ & $\begin{array}{l}\text { Jay D. } \\
\text { Amsterdam, } \\
\text { Justine hults, } \\
\text { Irene Soeller, } \\
\text { Jun J. Mao, } \\
\text { Kenneth } \\
\text { Rockwell and } \\
\text { Andrew B. } \\
\text { Newberg }\end{array}$ & 2012 & $\begin{array}{l}\text { Examinar a ação } \\
\text { antidepressiva do extrato } \\
\text { de camomila oral em } \\
\text { indivíduos com sintomas } \\
\text { de ansiedade e } \\
\text { depressão co-mórbidas. }\end{array}$ & $\begin{array}{c}\text { Estudo com } 57 \text { indivíduos ansiosos } \\
\text { com ou sem história pregressa ou } \\
\text { atual de depressão, que receberam } \\
\text { extrato de camomila ou placebo. } \\
\text { Foram analisadas mudanças clínicas a } \\
\text { partir da Escala de Depressão de } \\
\text { Hamilton }\end{array}$ \\
\hline $\begin{array}{l}\text { A randomized, double- } \\
\text { blind, placebo- } \\
\text { controlled trial of oral } \\
\text { Matricaria recutita } \\
\text { (chamomile) extract } \\
\text { therapy of generalized } \\
\text { anxiety disorder }\end{array}$ & $\begin{array}{l}\text { Jay D. } \\
\text { Amsterdam, } \\
\text { Yimei Li, } \\
\text { Irene } \\
\text { Soeller, } \\
\text { Kenneth } \\
\text { Rockwell, } \\
\text { Jun J. Mao } \\
\text { and Justine } \\
\text { Shults }\end{array}$ & 2009 & $\begin{array}{l}\text { Avaliar a hipótese de a } \\
\text { camomila ser superior ao } \\
\text { placebo na redução dos } \\
\text { sintomas de TAG com } \\
\text { um perfil de } \\
\text { tolerabilidade } \\
\text { comparável. }\end{array}$ & $\begin{array}{l}\text { Estudo randomizado, duplo-cego, } \\
\text { controlado por placebo sobre eficácia e } \\
\text { tolerabilidade do extrato de Matricaria } \\
\text { recutita (camomila) em pacientes com } \\
\text { transtorno de ansiedade generalizada } \\
\text { leve a moderada (TAG). }\end{array}$ \\
\hline
\end{tabular}

Fonte: Autoria própria (2019) 


\subsection{Análise da terapia com camomila em indivíduos com tag e/ou sintomas depressivos}

A camomila, sob a forma de chás e óleos, é frequentemente utilizada devido seu efeito calmante. Sendo seu mecanismo de ação ainda desconhecido. No entanto, várias linhas de evidência sugerem que um ou mais dos seus constituintes flavonóides podem produzir atividade ansiolítica afetando o GABA, noradrenalina (NA), dopamina (DA) e neurotransmissão de serotonina ou modulando a função do eixo hipotálamo-hipófiseadrenocortical (AMSTERDAM et al., 2009). A apigenina e outros constituintes da camomila, também se mostraram capazes de se ligar aos receptores benzodiazepínicos e reduzir a atividade ativada pelo GABA (MAO et al., 2014).

De acordo com o estudo realizado por Keefe et al (2016), constatou-se uma resposta clinicamente significativa $(58,1 \%$ dos indivíduos) à terapia com a camomila durante oito semanas de TTO. A dosagem utilizada durante o período, $1500 \mathrm{mg}$ (três cápsulas de $500 \mathrm{mg}$ diárias) foi bem tolerada e sem eventos adversos graves relatados.

No estudo de Amsterdam et. al (2012), foi analisada a atividade antidepressiva da Matricaria recutita através da Escala de Depressão de Hamilton (HAM-D), os indivíduos que participaram da pesquisa foram adstritos em subgrupos: depressão co-mórbida atual, história pregressa de depressão, ou ausência de depressão atual ou passada. Observandose uma redução significativa em longo prazo nos escores totais do HAM-D, salientando a redução desses escores em indivíduos com depressão e ansiedade co-mórbidas. Embora os resultados tenham sido positivos, ensaios posteriores deverão ser executados para uma validação concreta das propriedades antidepressivas da Matricaria recutita.

\subsection{Vantagens do tratamento alternativo com a camomila}

Em estudos toxicológicos não clínicos referidos pelo Ministério da Saúde em "MONOGRAFIA DA ESPÉCIE Matricaria chamomilla L. (= Chamomilla recutita (L.) Rauschert, CAMOMILA)" (2015), demonstraram que derivados vegetais da Matricaria recutita não apresentaram toxicidade em doses testadas em camundongos, inexistindo sinais como excitação, tremores, contrações musculares, descoordenação motora, reflexo de endireitamento e alterações respiratórias, nem tão pouco óbitos.

Foram avaliadas as toxicidades aguda, subcrônica e crônica em diversas apresentações, como óleo essencial, extrato hidroalcoólico/etanólico, extrato metanólico e extrato líquido. A dose letal 50\% (DL50) do extrato foi maior do que 10.000 mg de extrato 
kg-1 de peso corporal, demonstrando assim confiabilidade na segurança de uso terapêutico da matricaria recutita, tornando-a uma válida alternativa no TTO do TAG pela baixíssima toxicidade (BRASIL, 2015).

O extrato de camomila produziu uma redução clinicamente significativa em pacientes com TAG moderado a grave. O controle foi feito através das Escala de Ansiedade de Hamilton, do Inventário de Ansiedade de Beck e do Índice Geral de Bem-estar Psicológico. Os resultados foram comparáveis com a terapia com drogas ansiolíticas convencionais, porém com menores índices eventos adversos (KEEFE et al., 2016).

\section{CONCLUSÃO}

O uso de plantas medicinais vem ganhando espaço no que se refere a alternativas terapêuticas, observado o número de estudos que vem sendo feitos para a testagem do potencial terapêutico de novos extratos. No contexto dos tratamentos ansiolíticos, observou-se com o presente estudo que a Matricaria recutita tem destaque relevante, apresentando resultados satisfatórios no controle da ansiedade, bem como na redução dos sintomas depressivos, além de chamar a atenção por sua baixa toxicidade, o que representa importante característica para a produção de um fitoterápico, de forma a abranger seu uso para além empirismo empregado pela cultura popular, o que proporcionaria maior aproveitamento de sua propriedades farmacológicas.

\section{REFERÊNCIAS}

AMERICAN PSYCHIATRIC ASSOCIATION (Estados Unidos da América). Manual diagnóstico e estatístico de transtornos mentais: DSM-5. 5. ed. Porto Alegre: Artmed, 2014.

AMSTERDAM, J.D. et al. A randomized, double-blind, placebo-controlled trial of oral Matricaria recutita (chamomile) extract therapy of generalized anxiety disorder. Journal of clinical psychopharmacology, v. 29, n. 4, p. 378, 2009.

AMSTERDAM, J.D. et al. Chamomile (matricaria recutita) may have antidepressant activity in anxious depressed humans-an exploratory study. Alternative therapies in health and medicine, v. 18, n. 5, p. 44, 2012. 
ANDREATINI, R.; BOERNGEN-LACERDA, R.; ZORZETTO FILHO, D. Pharmacological treatment of eneralized anxiety disorder: future perspectives. Revista Brasileira de Psiquiatria, v. 23, n. 4, p. 233-242, 2001.

BRASIL. Agência Nacional de Vigilância Sanitária. Formulário de Fitoterápicos da Farmacopéia Brasileira / Agência Nacional de Vigilância Sanitária. Brasília: Anvisa, 2011.

BRASIL. Farmacopeia Brasileira, volume 1 / Agência Nacional de Vigilância Sanitária. Brasília: Anvisa, 2010.

BRASIL. Ministério da Saúde. Secretaria de Ciência, Tecnologia e Insumos Estratégicos. Departamento de Assistência Farmacêutica. Política e Programa Nacional de Plantas Medicinais e Fitoterápicos / Ministério da Saúde, Secretaria de Ciência, Tecnologia e Insumos Estratégicos, Departamento de Assistência Farmacêutica. - Brasília: Ministério da Saúde, 2016.

BRASIL. MONOGRAFIA DA ESPÉCIE Matricaria chamomilla L. (= Chamomilla recutita (L.) Rauschert, CAMOMILA). Ministério da Saúde / ANVISA: Brasília, 2015.

BRASIL. RESOLUÇÃO DA DIRETORIA COLEGIADA - RDC № 17, DE 24 DE FEVEREIRO DE 2000 / AGÊNCIA NACIONAL DE VIGILÂNCIA SANITÁRIA.

Brasília: ANVISA, 2000.

BRASIL. RESOLUÇÃO DA DIRETORIA COLEGIADA - RDC № 49, DE 23 DE NOVEMBRO DE 2010 / AGÊNCIA NACIONAL DE VIGILÂNCIA SANITÁRIA.

Brasília: ANVISA, 2010.

FAUSTINO, T.T.; ALMEIDA, R.B.; ANDREATINI, R. Plantas medicinais no tratamento do transtorno de ansiedade generalizada: uma revisão dos estudos clínicos controlados. Revista Brasileira de Psiquiatria, v. 32, n. 4, p. 429-36, 2010.

GOODMAN \& GILMAN. As bases farmacológicas da terapêutica. 10ª . ed. Rio de Janeiro: McGraw-Hill, 2005. 
KEEFE, J.R. et al. Short-term open-label chamomile (Matricaria chamomilla L.) therapy of moderate to severe generalized anxiety disorder. Phytomedicine, v. 23, n. 14, p. 1699-1705, 2016.

LARANJEIRA, R.; CASTRO, L.A. Potencial de abuso de benzodiazepínicos. In: Bernik MA editor. Benzodiazepínicos, quatro décadas de experiência. São Paulo (SP): Edusp, 1999.

MAO, J.J. et al. Long-term chamomile (Matricaria chamomilla L.) treatment for generalized anxiety disorder: A randomized clinical trial. Phytomedicine, v. 23, n. 14, p. 1735-1742, 2014.

SADOCK, B.J.; SADOCK, V.A.; RUIZ, P. Compêndio de Psiquiatria: Ciência do Comportamento e Psiquiatria Clínica. 11. ed. Porto Alegre: Artmed, 2017.

SILVEIRA, P.R. Fundamentos da psiquiatria. Rio de Janeiro: Lumen Juris, 2014. SIMÕES, C.M.O. et. al. Farmacognosia: da planta ao medicamento. 6 ed. Porto Alegre: Editora da UFRGS; Florianópolis: Editora da UFSC, 2007.

STAHL, S.M. Psicofarmacologia: bases neurocientíficas e aplicações práticas.

4. Ed. Rio de Janeiro: Guanabara Koogan, 2014. 